\title{
Revisión sistemática: incidencia de reacciones cutáneas a las drogas
}

Rates of Cutaneous Reactions to Drugs. Bigby M. Arch Dermatol 2001; 137:765-770.

\section{Objetivo}

Determinar la validez, magnitud, precisión y aplicabilidad de los datos de las tasas de incidencia de las reacciones adversas cutáneas causadas por drogas.

\section{Diseño}

Revisión sistemática de la literatura médica.

\section{Lugar}

Departamento de Dermatología, Escuela de Medicina de Harvard y centro Médico Beth Israel, Boston, EE.UU.

\section{Fuente de Datos}

Se realizó una búsqueda en la base de datos de MEDLINE (1966Agosto 2000) para estudios que contenían información sobre la incidencia de reacciones cutáneas a las drogas en adultos y niños. Se examinaron las bibliografías de los artículos y revisiones encontradas para buscar otros estudios relevantes.

\section{Medición de resultados principales}

Se evaluó la validez, magnitud, precisión y aplicabilidad de los estudios que incluyeron datos sobre la incidencia de reacciones cutáneas; utilizando guías para la evaluación de los artículos acerca del daño y el pronóstico.

\section{Resultados}

Nueve estudios cumplieron con los criterios de inclusión. Cinco se basaron en datos prospectivos de pacientes internados, 2 fueron retrospectivos basados en la revisión de las historias clínicas de papel o computarizadas, y 2 en los reportes espontáneos y datos de consumo. Los estudios oscilaron entre 440 y 37.665 pacientes.
El exantema morbiliforme y la urticaria fueron las reacciones cutáneas más comunes a las drogas. La incidencia de las reacciones variaron de $0 \%$ a $8 \%$ en las distintas familias de drogas, y fueron más altas para los antibióticos (rango de $1 \%$ a $8 \%$ para varias clases de antibióticos). Muchas drogas fueron administradas a más de mil pacientes y no presentaron en ellos una reacción adversa cutánea (ej. paracetamol, digoxina, aspirina, metildopa, codeina, espironolactona, propranolol, warfarina, hidroclorotiazida, hidrocortisona). Los antiinflamatorios no esteroideos en distintos estudios presentaron una incidencia menor al $1 \%$

Reacciones cutáneas alérgicas (exantema morbiliforme y urticaria) a drogas recibidas en al menos 1000 pacientes $\left(\mathrm{BCDSP}^{\star}\right)$

\begin{tabular}{l|l|l}
\hline Droga & Tasa \% & IC95\% \\
\hline Amoxicilina & 5,1 & $3.9-6.4$ \\
\hline Ampicilina & 4,5 & $3.9-5.1$ \\
\hline Cotrimoxazol & 3,7 & $2.7-4.8$ \\
\hline Penicilinas semisintéticas & 2,9 & $2.0-3.7$ \\
\hline Glóbulos rojos & 2,0 & $1.5-2.4$ \\
\hline Penicilina G & 1,6 & $1.2-2.0$ \\
\hline Cefalosporinas & 1,5 & $0.9-2.1$ \\
\hline Gentamicina & 1,0 & $0.5-1.6$ \\
\hline
\end{tabular}

*BCDSP indica Boston Collaborative Drug Surveillance Programe

\section{Conclusiones}

Las manifestaciones más frecuentes son el exantema morbiliforme y la urticaria. El grupo con la mayor tasa de reacciones adversas cutáneas es el de los antibióticos. Los antiinflamatorios no esteroideos presentaron tasas menores.

\section{Comentario}

A pesar de las diferencias metodológicas y de los momentos de realización de estos estudios, existe acuerdo en sus resultados. Las reacciones cutáneas más frecuentes fueron el exantema morbiliforme y la urticaria. Fueron más observadas con los antibióticos y luego con los antiinflamatorios no esteroideos. No existen grandes diferencias entre los distintos grupos etarios y el género al que pertenecen. Algunas criticas podrían realizarse: faltarían datos sobre la relación entre drogas, comorbidades; y datos acerca de la influencia de la polimedicación con estas reacciones.

A pesar de presentar criterios para analizar la validez, magnitud y precision de los estudios este trabajo fue realizado por un solo autor, por lo tanto deberíamos estar atentos a algunos sesgos*; especialmente en la selección (sesgo de selección*) y en la medición de la calidad de los estudios.

Deberíamos recordar también el sesgo de publicación ya que solo se utilizaron estudios publicados en MEDLINE.

Por otro lado esta es la única revisión publicada en los ultimos 10 años con lo cual no es posible realizar ningún tipo de comparación con otras publicaciones y parece ser la mejor información con la que contamos. Considero que algunos puntos interesantes podrían agregarle mayor utilidad a esta revisión para nuestra práctica cotidiana, por ejemplo, datos sobre las interacciones con otras drogas, la relación con las dosis administradas (¿a más dosis más probabilidad de reacción adversa?) y por último, la relación con las distintas comorbilidades. Esperemos que pronto hayan nuevos resultados para contestarnos estas preguntas. 